\title{
Dielectric Barrier Discharge Plasma Actuator for Load Alleviation and Instability Control in a Compressor Cascade
}

\author{
Maria Grazia De Giorgi ${ }^{1,{ }^{*},}$ Antonio Suma ${ }^{1}$, Alessia Laforì $^{1}$ and Antonio Ficarella ${ }^{1}$ \\ ${ }^{1}$ University of Salento, Department of Engineering of Innovation, 73100 Lecce, Italy \\ *mariagrazia.degiorgi@unisalento.it
}

\begin{abstract}
The aim of this study is to investigate the performance of dielectric barrier discharge plasma actuators (DBD-PAs) applied to control the aeroelastic response of a compressor cascade in subsonic flow conditions. Simulations involve a cascade composed by 7-blade, 3 of which show a not-synchronous pitching behaviour. Two different inter blade phase angle (IBPA) have been tested and compared. Actuators capabilities in terms of load alleviation and instability control have been evaluated through the measurement of mean value, standard deviation and hysteresis area of the airfoil response in terms of lift and moment coefficients.
\end{abstract}

\section{Introduction}

Aeroelastic phenomena have been long investigated, since they represent severe issues for structural integrity of many aerospace applications [1]. Analysis and prediction of aeroelastic mechanisms are still a major topic of research, due to their complexity and number of variables involved. In accordance with modern design trends, blades are made slender and longer and, hence, more likely susceptible to flutter when subjected to unsteady dynamic loads. Flutter is a self-excited and self-sustained aeroelastic instability that occurs due to the interaction between aerodynamic, inertial and elastic forces acting on the blade [2]. Because of the coupling of the flexural and torsional modes of vibration, the blades can experience both flapwise deflections and twist. Moreover, when the airflow passing over the blade provides a periodic frequency near or equal to the blade natural one, resonance phenomenon takes place. Blade vibrations are, then, violently amplified and can no longer be compensated by the structural damping [3] [4]. This results in the immediate loss of the blade or in high cycle fatigue failure (HCF) in the long term. Nowadays, different technologies are available to manipulate the flow [5], such as plasma actuators. These devices induce a local speed perturbation in a region next to the blade surface [6] [7]. The advantages of plasma actuators include high dynamic responses, light weight, construction simplicity, absence of moving parts, low power required, easy installation and compatibility with already existing aerodynamic surfaces [8]. Therefore, the purpose of this paper is to evaluate the effects of these devices during critical conditions, in order to improve compressor efficiency.

\section{Computational Model}

The tests involve a 2D cascade composed by seven NACA65 blades. The foil is located about $2 \mathrm{c}$ from the inlet wall. Each blade row has a mean angle of attack already set to $\alpha_{0}=$ 
$2^{\circ}$, and a chord length $\mathrm{c}=0.15 \mathrm{~m}$. Actuation devices are placed at the trailing edge of the three central blades, indexed with the number 3-4-5.

A 506870 cells mesh reproduces the entire domain. Boundary layer profile, with a dimensionless wall distance $y^{+}=0.924$, is resolved by the O-grid wrapped around the airfoil. A quad-map meshing scheme was chosen to mesh each face. Fig. 1 shows a detail of the grid around the leading and trailing edge of the blade. Numerical simulations are performed for two freestream velocities imposed at the inlet $\mathrm{U}_{\infty}=34.36 \mathrm{~m} / \mathrm{s}$ and $\mathrm{U}_{\infty}=19.65 \mathrm{~m} / \mathrm{s}$, giving a Reynolds number equal to $\operatorname{Re} \approx 3 \cdot 10^{5}$ and $\operatorname{Re} \approx 2 \cdot 10^{5}$ respectively. In accordance with experiments described in Refs. [9] , [10], the outlet static pressure is set as atmospheric pressure $(101325 \mathrm{~Pa})$, while periodic boundary conditions are specified for the top and bottom wall of the domain. To assess the efficiency and accuracy of the reference grid, a sensitivity study was carried out and discussed later in detail.
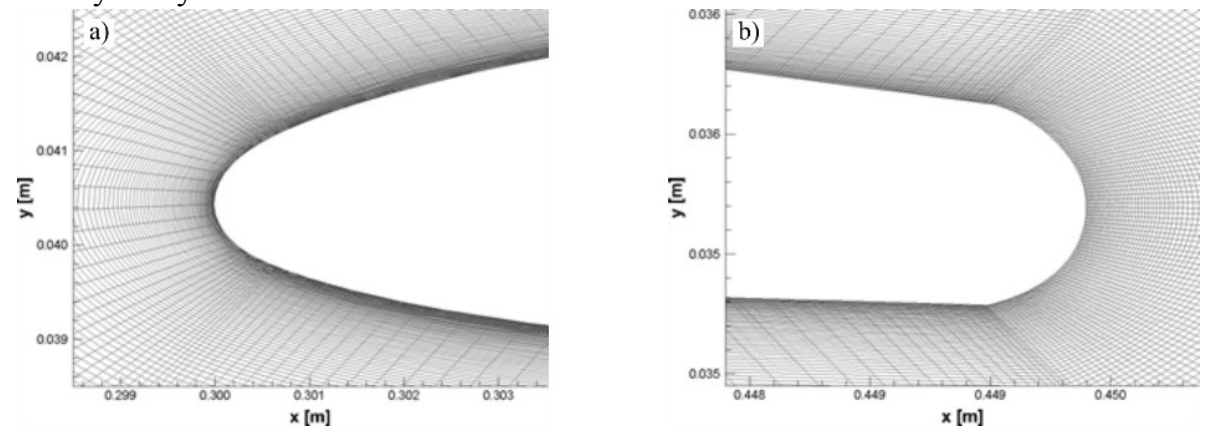

Fig. 1. Detail of the grid of the blade: (a) leading edge and (b) trailing edge

The finite-volume solver Ansys FLUENT ${ }^{\circledR}$ (release 19.2) is used for the present work. A simple second-order upwind scheme discretization is selected for the transient formulation and k- $\omega$ SST viscous model with a pressure-based approach is employed. The basic k- $\omega$ model better solves boundary layer problems with a near-wall resolution at low Reynolds. While, the shear-stress transport model (SST) help to overcome deficiencies of the k- $\omega$ in the near-wall regions. So, neglecting the three-dimensional effects of the flow, all incompressible simulations were performed with the following properties: fluid is air with density $\rho=1.225$ $\mathrm{kg} / \mathrm{m}^{3}$, dynamic viscosity is equal to $\mu=1.80210^{-5} \mathrm{~kg} / \mathrm{m} \mathrm{s}$, ratio of specific heats is $\mathrm{cp} / \mathrm{cv}=$ 1.4 , reference temperature and pressure of $15^{\circ} \mathrm{C}$ and $101325 \mathrm{~Pa}$ respectively. The diffusionbased smoothing method was selected to adapt the mesh when nodes near walls are moving. Furthermore, no-slip boundary conditions were imposed on the airfoils surfaces. Convergence criteria of $10^{-6}$ were set and the steady-state solution was used as initial condition for the time-domain computations.

An angular displacement was applied to the three central blades in order to simulate the changing in the incidence angle with time. The displacement was dependent of the number of the airfoil, following the law:

$$
\alpha(t)=\alpha_{0}+\bar{\alpha} \cdot \sin (2 \pi f \cdot t+n \cdot I B P A)
$$

where $\alpha(t)$ is the angle of attack measured clockwise, $\alpha_{0}$ is the mean incidence angle and $n$ is the index of the airfoil. The center of rotation is the mid-chord of the airfoil.

\section{Plasma actuator modelling}

Dielectric Barrier Discharge Plasma Actuator (DBD-PA) consists of two metallic electrodes separated by a dielectric barrier (Fig. 2). Electrodes are arranged asymmetrically, one is exposed to the free flow, while the other is embedded in a dielectric material. The actuator induces a local flow perturbation, that modifies the boundary layer velocity profile 
over the blade surface [11], [12]. The exposed electrode is fed by a high voltage electric signal, the other one is grounded. The electric field between electrodes brings to the generation of a non-thermal plasma, producing thus an electro-dynamic force proportional to the charge density and the electric field strength. For this study, the actuator experimentally studied by Thomas et al. [13] has been reproduced, in terms of generated body force, by using a simple triangular time-averaged body force model such as proposed by Shyy et al. [14].
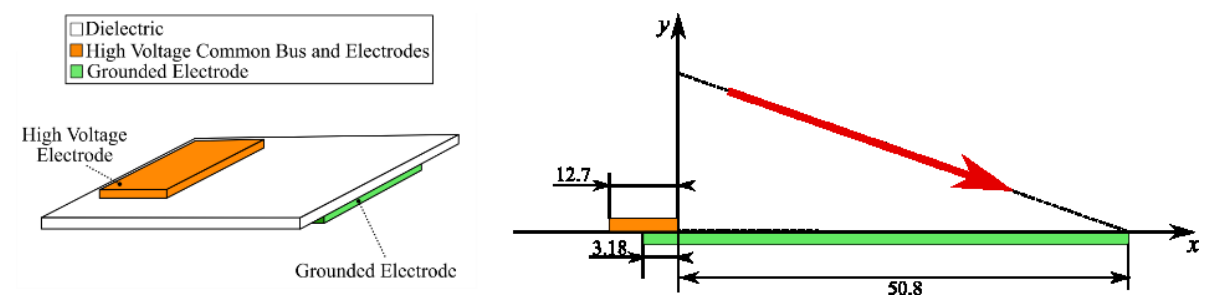

Fig. 2. Schematic of DBD actuator with generated force indication

The body force field produced by the actuator was approximated as a triangular timeaveraged force distribution, using a linear expression, as described in [14]:

$$
\overrightarrow{\mathrm{F}}(\mathrm{x}, \mathrm{y})=\left(|\mathrm{F}| \frac{\mathrm{k}_{2}}{\sqrt{\mathrm{k}_{1}^{2}+\mathrm{k}_{2}^{2}}},|\mathrm{~F}| \frac{\mathrm{k}_{1}}{\sqrt{\mathrm{k}_{1}^{2}+\mathrm{k}_{2}^{2}}}\right)
$$

where $|\mathrm{F}|=\left|\mathrm{F}_{0}-\mathrm{k}_{1} \mathrm{x}-\mathrm{k}_{2} \mathrm{y}\right|$, and $(\mathrm{x}, \mathrm{y})$ is the actuator local coordinate system. The constants $\mathrm{F}_{0}, \mathrm{k}_{1}$ and $\mathrm{k}_{2}$ were numerically calibrated to match the experimentally velocity profiles on a flat plate reported in [13]. $F_{0}$ is the value of the force at the origin of the local coordinate axis, which starts at the end of the exposed electrode, with the $x$ axis aligned with the actuator. The coefficients $\mathrm{k}_{1}$ and $\mathrm{k}_{2}$, instead, are defined to limit the body force field in a triangular region with a height $\mathrm{a}=15 \mathrm{~mm}$ and a width $\mathrm{b}=50.8 \mathrm{~mm}$. In particular, $\mathrm{k}_{1}=$ $F_{0} / b$ while $\mathrm{k}_{2}=F_{0} / a$. The resulting body-force field was included into the CFD computation by introducing a new term $(F / \rho)$ into the momentum equation of the NavierStokes system for an incompressible flow:

$$
\left\{\begin{array}{c}
\nabla \cdot \mathrm{U}=0 \\
\frac{\partial \mathrm{U}}{\partial \mathrm{t}}+\nabla \cdot(\mathrm{UU})-\nabla \cdot(\mu \cdot \nabla \mathrm{U})=\frac{\mathrm{F}}{\rho}-\frac{\nabla \mathrm{p}}{\rho}
\end{array}\right.
$$

\section{Numerical Validation}

\subsection{Steady aerodynamics}

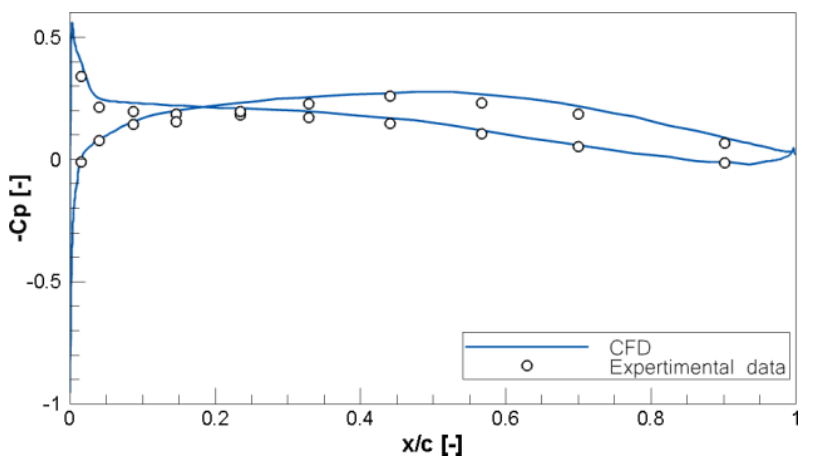

Fig. 3. Cp distribution of the central blade for the clean case; $\alpha_{0}=2{ }^{\circ}, \mathrm{U}_{\infty}=34.36 \mathrm{~m} / \mathrm{s}, \mathrm{Re}=350000$. 
Convergence assessment of the reference grid was realized by running a steady-state simulation, for the rough, medium and finer mesh. Computations are performed $\alpha_{0}=2^{\circ}$ and $\mathrm{U}_{\infty}=34.36 \mathrm{~m} / \mathrm{s}$ that gives a Reynolds $\mathrm{Re}=350000$ and a flow Mach number $\mathrm{M}_{\infty}=0.1$. Chordwise pressure distribution for the clean configuration of the central blade $(n=4)$ are compared to experimental data obtained on the test rig at TU Berlin (Fig. 3). As shown, there is a very good agreement between the computed and the experimental performances. Aerodynamic coefficients estimated on the reference grid are presented as percent deviation from the rough, medium and finer distribution results.

Table 1 shows that differences in loads are always below $1 \%$ and, hence, no further refinement of the grid is required. An accurate estimation of the drag coefficient $C_{D}$ is not a principal objective of this study, because the flow was assumed to be fully turbulent and RANS computations were used. Rough and finer mesh predict similar results, consistently with [15], [16] and [17]. This comparison shows that a mesh independent solution has been achieved and results are accurate. In conclusion, the reference grid can be considered the best compromise solution between accuracy, computational load for the whole cascade.

Table 1. Mesh independency results: aerodynamic loads computed using the reference grid and the same parameters measured on the rough, medium and finer mesh.

$\begin{array}{cccc}\text { Grid } & \Delta \mathrm{C}_{\mathrm{L}} \% & \Delta \mathrm{C}_{\mathrm{D}} \% & \Delta \mathrm{C}_{\mathrm{M}, \mathrm{c} / 2} \% \\ \text { Reference/ rough } & 0.08 & -0.33 & 0.09 \\ \text { Reference/medium } & 0.14 & -0.54 & -0.11 \\ \text { Reference/finer } & 0.2 & -0.74 & -0.28\end{array}$

\subsection{Unsteady aerodynamics}

A forced oscillation is imposed to the central blades (index 3, 4, 5) with the purpose of validating the solver set-up and the transient dynamics of the reference grid. Experimental results from [18], [19] [20] are employed for this validation. The time-domain flow simulation of the pitching motion is performed under the following conditions: $\alpha_{0}=2^{\circ}, \bar{\alpha}=1^{\circ}$, inlet velocity $\mathrm{U}_{\infty}=34.36 \mathrm{~m} / \mathrm{s}, \mathrm{IBPA}=0^{\circ}$ and reduced frequency $\mathrm{k}=2 \pi \mathrm{f} \mathrm{c} / \mathrm{U}_{\infty}=0.144$. The center of rotation is set to the mid-chord location of each blade, consistently with experimental. Three periods are simulated to ensure convergence.

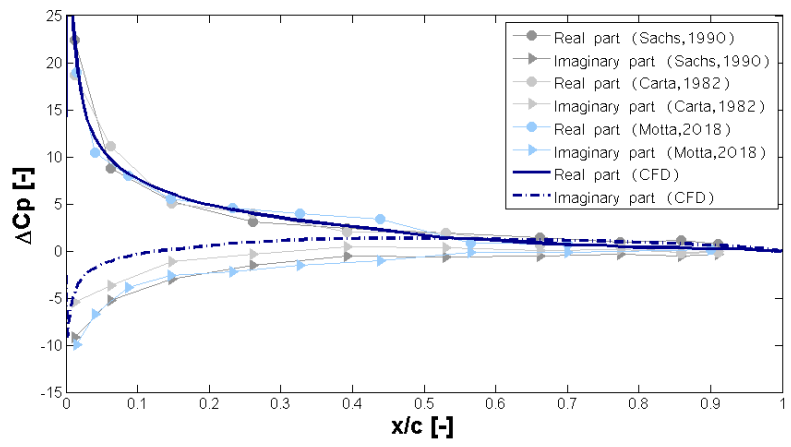

Fig. 4. Unsteady $\Delta \mathrm{Cp}$ of the 4 th blade for $\mathrm{U}_{\infty}=34.36 \mathrm{~m} / \mathrm{s}, \mathrm{f}=5.25 \mathrm{~Hz}, \mathrm{Re}=350000$ and $\mathrm{IBPA}=0^{\circ}$.

Complex-valued pressure coefficient $\mathrm{Cp}$ is found by taking the fast Fourier transform (FFT) of the blade pressure distribution for both the suction and pressure side, and is defined as follows:

$$
C_{p}=\frac{p(x, t)}{q \cdot \bar{\alpha}}
$$

Where $\mathrm{p}(\mathrm{x}, \mathrm{t})$ is the surface static pressure of the blade, $\bar{\alpha}=1^{\circ}$ is the torsional amplitude and $q=(1 / 2) \rho U^{2}$ is the inlet dynamic pressure. Real and imaginary part of the pressure 
distribution $\mathrm{p}(\mathrm{x}, \mathrm{t})$ are calculated individually for the PS and SS of the central blade $(\mathrm{n}=4)$.

The unsteady differential pressure coefficient $\Delta \mathrm{Cp}$ is given by Eq. (5):

$$
\Delta C_{p}=C_{p, l o w}-C_{p, \text { up }}
$$

Where the subscripts low and up refer respectively to the lower (pressure) and upper (suction) surface of the airfoil blade. Fig. 4 presents the unsteady surface pressure distribution on the 4 th blade oscillating in pitching mode.

\section{Actuation Time-Domain Analysis}

Transient simulations of the compressor cascade equipped with DBD plasma actuators involve a subsonic flow with a freestream Mach number $\mathrm{M}=0.057$ and inlet velocity set to $\mathrm{U}_{\infty}=19.65 \mathrm{~m} / \mathrm{s}$, which gives a Reynolds number $\mathrm{Re}=200000$. In this case, a reduced frequency of $\mathrm{k}=0.919$, pitching amplitude $\bar{\alpha}=1^{\circ}$ and frequency $\mathrm{f}=19.17 \mathrm{~Hz}$ are imposed to the blades. In order to assess the effectiveness of the DBD actuators, the IBPAs of $90^{\circ}$ and $51.43^{\circ}$ have been tested. This because a significant increase of the vibratory load was observed both numerically at IBPA $=51.43^{\circ}[9]$ and experimentally at IBPA $=51^{\circ}[10]$. Three periods have been simulated to ensure the convergence of the solution for each case.
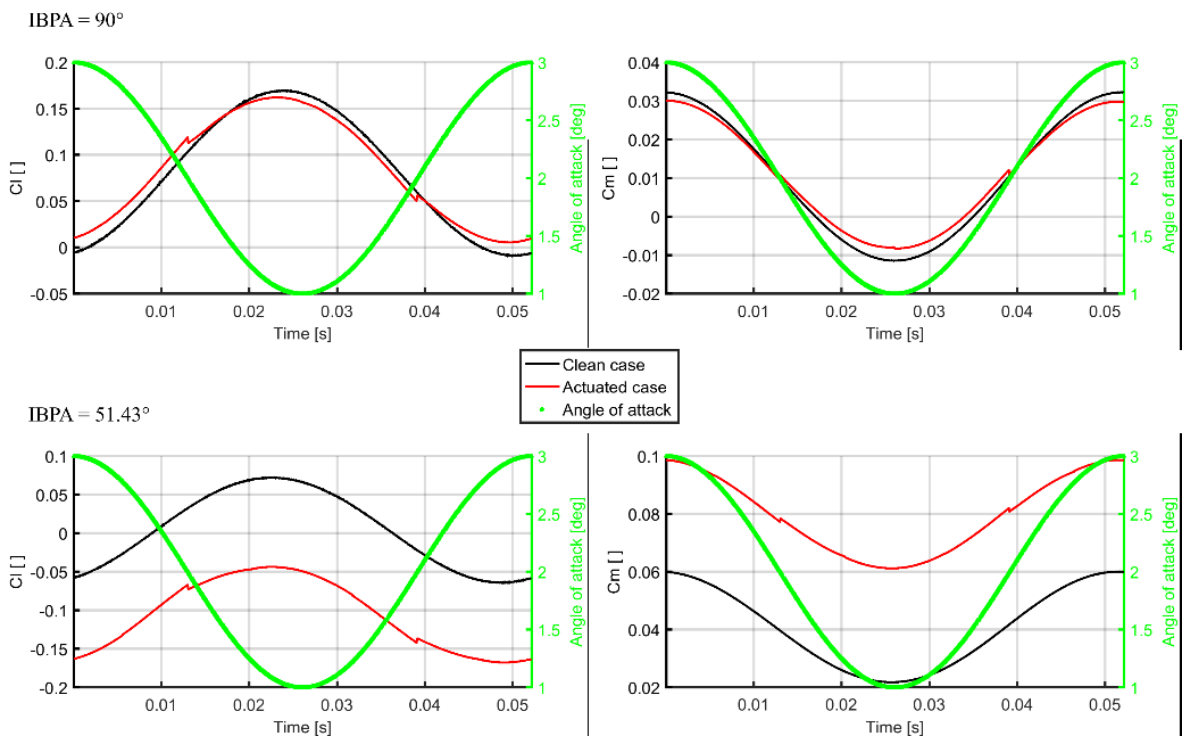

Fig. 5. $\mathrm{C}_{\mathrm{L}}$ and $\mathrm{C}_{\mathrm{M}}$ for the cases with $\mathrm{IBPA}=90^{\circ}$ (up) and $51.43^{\circ}$ (down). Clean and actuated cases.

The upper and lower actuators are activated alternately. The first of them has the capability to increase the load locally in the actuation region, while the other one decreases the load. The upper actuator is activated when the angle of attack is greater than its mean value $\alpha_{0}=2^{\circ}$, while in the other case the device located on the pressure surface is activated.

This modified performance of the airfoil can be easily monitored by measuring mean value and standard deviation of the lift and moment coefficients signals.

Table 2, the mean value of the clean case lift coefficient is increased by the presence of actuation only in the case of IBPA $=90^{\circ}$, while it is reduced in the case with IBPA $=51.43^{\circ}$. The moment coefficient mean value, instead, is increased in all the cases, suggesting an increment of the nose-down component of the torque. The standard deviation of the signal is reduced in all the cases, underlining the alleviation capabilities of the DBD. 
Table 2. Load alleviation results.

\begin{tabular}{ccccccc|} 
& \multicolumn{3}{c}{ IBPA $=90^{\circ}$} & \multicolumn{3}{c}{ IBPA $=51.43^{\circ}$} \\
& Clean & Actuated & Variation & Clean & Actuated & Variation \\
C $_{L}$ mean value & 0.08098 & 0.08412 & $+3.87 \%$ & 0.00478 & -0.10535 & $-2303 \%$ \\
\hline CL standard deviation & 0.06295 & 0.05542 & $-11.96 \%$ & 0.04808 & 0.04474 & $-6.95 \%$ \\
CM mean value & 0.01033 & 0.01090 & $+5.54 \%$ & 0.04083 & 0.07980 & $+95.43 \%$ \\
\hline C $_{M}$ standard deviation & 0.01544 & 0.01355 & $-12.22 \%$ & 0.01351 & 0.01305 & $-3.35 \%$
\end{tabular}

Pitching airfoils show a phase lag between the instantaneous angle of attack and the aerodynamic response of the airfoil, causing a hysteresis in the curve lift coefficient/angle of attack or moment coefficient/angle of attack. The area of the hysteresis loop of the moment coefficient, during a pitching period, represents the work done on the flow by the profile. If the hysteresis area is positive, the profile works on the flow, having a damping effect on the profile motion. Instead, if the area is negative, the flow works on the profile, rising the profile energy and bringing to increasingly large oscillations [21]. Moreover, an increasing hysteresis can induce the developing of dynamic stall on the profile [22] [23].

$$
\text { Area } \mathrm{H}_{C_{M}}=-\int_{\alpha_{\max , \mathrm{i}}}^{\alpha_{\max \mathrm{i}+1}} \mathrm{C}_{M, c / 2} \cdot d \alpha
$$
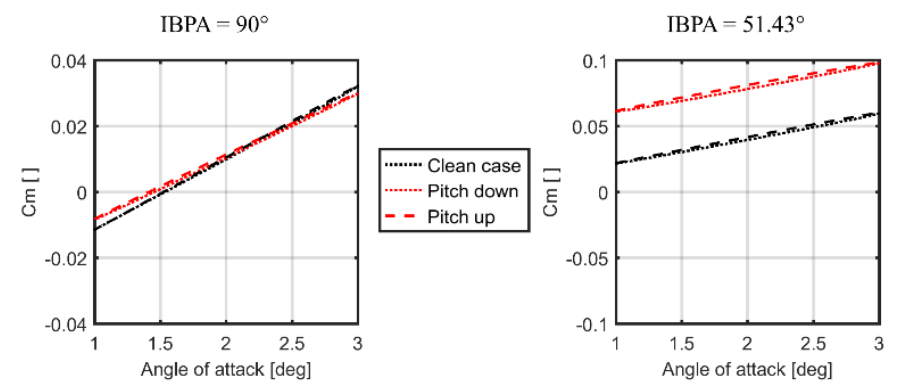

Fig. 6. $\mathrm{CM}_{\mathrm{M}}$ hysteresis curve for the cases with IBPA $=90^{\circ}$ (left) and IBPA $=51.43^{\circ}$ (right).

As shown in Fig. 6, the hysteresis area of the moment coefficient of the clean cases has an almost zero value, meaning a little energy flux exchanged between aerodynamic profile and flow. The presence of actuation leads to not relevant changes either in shape either in the value of the hysteresis area.

\section{Conclusion}

In the present work, numerical analyses were conducted to study the applicability of using plasma actuators to modify the local flow around the trailing edge of the compressor blades.

A reduction of the unsteady peaks on both pitching lift and moment signal is observed in all the analysed cases, as shown by the signal standard deviation. The moment coefficient mean value is increased by the unsteady application of DBDs either for the case with IBPA $=51.43^{\circ}$, either for the case with IBPA $=90^{\circ}$. Moreover, a small lift enhancement has been achieved in the case with IBPA $=90^{\circ}$. The force applied by the simulated actuator, results enough to lead to modification of the airfoil response in terms load alleviation. A smaller device, applied to a region next to the trailing edge, could increase the performance in terms of moment coefficient control and stability improvement of the airfoil. 


\section{Nomenclature}

$\alpha \quad$ angle of attack [deg]

$\alpha_{0}$ mean angle of attack [deg]

$\mathrm{k}=2 \pi \mathrm{f} \mathrm{c} / \mathrm{U}_{\infty}$ reduced frequency $[\mathrm{Hz}]$

$\mathrm{M}_{\infty}$ freestream Mach number

$\bar{\alpha}$ oscillation amplitude [deg]

$\rho$ density $\left[\mathrm{kg} / \mathrm{m}^{3}\right]$

$\omega=2 \pi \mathrm{f}$ angular frequency $[\mathrm{rad} / \mathrm{s}]$

c blade chord length [m]

$\mathrm{C}_{\mathrm{D}}$ drag coefficient

$\mathrm{C}_{\mathrm{p}}$ pressure coefficient

$\mathrm{C}_{\mathrm{L}}$ lift coefficient

$\mathrm{C}_{\mathrm{M}, \mathrm{c} / 2}$ mid-chord moment coefficient

E electric field strength $[\mathrm{V} / \mathrm{m}]$

f frequency [Hz]

$\mathrm{F}$ body force $\left[\mathrm{N} / \mathrm{m}^{3}\right]$

$\mathrm{n} \mathrm{n}^{\text {th }}$ blade
Re Reynolds number

$t$ time [s]

$\mathrm{T}$ oscillation period $[\mathrm{s}]$

$\mathrm{U}_{\infty}$ freestream velocity $[\mathrm{m} / \mathrm{s}]$

$\mathrm{x}$ horizontal coordinate [m]

$\mathrm{y}$ vertical coordinate $[\mathrm{m}]$

$\mathrm{AC}$ alternating current

a.o.a. angle of attack

c.g. center of gravity

DBD dielectric barrier discharge

IBPA interblade phase angle [deg]

L.E. leading edge

PS pressure side

SS suction side

T.E. trailing edge

\section{References}

[1] R. Bisplinghoff, H. Ashley and R. Halfman, Aeroelasticity, New York, USA: Dover Publications, 1955.

[2] A. Zanotti, D. Grassi and G. Gibertini, "Experimental investigation of a triling edge Lshaped tab on a pitching airfoil in deep dynamic stall conditions," Proc IMechE Part G: J Aerospace Engineering, vol. 228, no. 12, pp. 2371-2382, 2014.

[3] F. Carta, "A Comparison of the Pitching and Plunging Response of an Oscillating Airfoil," Nasa Contractor Report 3172, 1979.

[4] C. Zhu and T. Wang, "Comparative Study of Dynamic Stall under Pitch Oscillation and Oscillating Freestream on Wind Turbine Airfoil and Blade," Applied Sciences, vol. 8, no. 1242, 2018.

[5] S. Sato, H. Yokoyama and A. Iida, "Control of Flow around an Oscillating Plate for Lift Enhancement by Plasma Actuators," Applied Sciences, vol. 9, no. 776, 2019.

[6] S. L. Cattafesta, "Actuators for Active Flow Control," Annual Review of Fluid Mechanics, vol. 43, pp. 247-272, 2011.

[7] M. G. De Giorgi, S. Traficante, C. De Luca, D. Bello and A. Ficarella, "Active Flow Control Techniques on a Stator Compressor Cascade: A Comparison Between Synthetic Jet and Plasma Actuators," in ASME Turbo Expo 2012: Turbine Technical Conference and Exposition, Copenhagen, Denmark, 2012.

[8] M. De Giorgi, D. Bello, S. Traficante and A. Ficarella, "Comparing Plasma Actuator Model and Application on a Compressor Cascade," in ISABE-2013-1105, 21th Isabe Conference, Busan, Korea, 2013.

[9] L. Malzacher, s. Geist, D. Peitsch and H. Hennings, "A low speed compressor test rig for flutter investigations," in Proceedings of ASME Turbo Expo 2016: Turbomachinery Technical Conference and Exposition, Seoul, South Korea, 2016. 
[10] H. Hennings, "Flutter Investigations on a Finite Linear 2D Compressor Cascade in a Wind Tunnel in Incompressible Flow," RWTH Aachen University, Aachen, Germany, 1997.

[11] E. Pescini, D. S. Martìnez, M. G. De Giorgi and A. Ficarella, “Optimization of micro single dielectric barrier discharge plasma actuator models based on experimental velocity and body force fields," Acta Astronautica, vol. 116, pp. 318-332, 2015.

[12] E. Pescini, F. Marra, M. G. De Giorgi, L. Francioso and A. Ficarella, "Investigation of the boundary layer characteristics for assessing the DBD plasma actuator control of the separated flow at low Reynolds numbers," Experimental Thermal and Fluid Science, vol. 81, pp. 482-498, 2017.

[13] O. Thomas, T. Corke, I. M., A. Kozlov and D. Schatzman, "Optimization of Dielectric Barrier Discharge Plasma Actuators for Active Aerodynamic Flow Control," AIAA Journal, vol. 47, no. 9, pp. 2169-2178, 2009.

[14] W. Shyy, B. Jayaraman and A. Andersson, "Modeling of glow discharge-induced fluid dynamics," Journal of Applied Physics, vol. 92, no. 11, pp. 6434-6443, 2002.

[15] V. Motta, L. Malzacher, P. Neumann and D. Peitsch, "Numerical assessment of virtual control surfaces for compressor blades," in 35th AIAA Applied Aerodynamics Conference, 59, June 2017.

[16] V. Motta, L. Malzacher and D. Peitsch, "Numerical investigation of virtual control surfaces for aeroelastic control on compressor blades," Journal of Fluids and Structures, vol. 81, pp. 617-637, 2018.

[17] V. Motta, L. Malzacher, V. Bicalho Civinelli de Almeida and D. Peitsch, "Aeroelastic control on compressor blades with virtual control surfaces: a numerical assessment," in Proceedings of ASME Turbo Expo 2018: Turbomachinery Technical Conference and Exposition, Oslo, Norway, 2018.

[18] C. F.O., "An Experimental Investigation of Gapwise Periodicity and Unsteady Aerodynamic Response in an Oscillating Cascade," NASA, United Technologies Research Center, 1982.

[19] V. Motta, L. Malzacher and D. Peitsch, "Numerical assessment of virtual control surfaces for load alleviation on compressor blades," Applied Sciences, vol. 8, no. 125, 2018.

[20] W. Sachs, "Windkanal fr Instationre Gitter (WiG), Messstrecke fr Instationre Gitter (MiG). Phase I: Bau und InbetriebnahmeWindkanal fr Instationre Gitter (WiG)," DLR, Institute of Aeroelasticity, Gttingen, Germany, 1990.

[21] M. McCroskey, "Unsteady Airfoils," Annual Review of Fluid Mechanics, vol. 14, no. 1, pp. 285-311, 1982.

[22] Q. Wang and Q. Zhao, "Rotor airfoil profile optimization for alleviating dynamic stall characteristics," Aerospace Science and Technology, vol. 72, pp. 502-515, 2018.

[23] A. Zanotti and G. Gibertini, "Experimental investigation of the dynamic stall phenomenon on a NACA23012 oscillating airfoil," Journal of Aerospace Engineering, vol. 0, pp. 1-14, 2012.

[24] M. Abdollahzadeh, J. Pascoa and P. Oliveira, "Comparison of DBD plasma actuators flow control authority in different modes of actuation," Aerospace Science and Technology, vol. 78, pp. 183-196, 2018. 